\title{
Side branch healing patterns of the Tryton dedicated bifurcation stent: a 1-year optical coherence tomography follow-up study
}

\author{
Maik J. Grundeken - Hector M. Garcia-Garcia - Robin P. Kraak • \\ P. Woudstra • Daniel M. de Bruin • Ton G. van Leeuwen • Karel T. Koch • \\ Jan G. Tijssen • Robbert J. de Winter • Joanna J. Wykrzykowska
}

Received: 27 March 2014/ Accepted: 18 July 2014/Published online: 26 July 2014

(C) Springer Science+Business Media Dordrecht 2014

\begin{abstract}
The bare-metal Tryton Side Branch (SB) Stent $^{\mathrm{TM}}$ (Tryton Medical, Durham, NC, USA) is used with a drug-eluting stent (DES) in the main branch (MB) to treat bifurcation lesions. It is argued that a drug-eluting Trytonversion is needed to improve clinical outcomes, although previous registries have shown good clinical results. More insights in neo-intimal hyperplasia (NIH) growth patterns of the Tryton treatment strategy are needed to decide if and where to drug-coat the stent. Ten patients returned for follow-up angiography (mean follow-up time $393 \pm 103$ days) and optical coherence tomography (OCT) pullbacks from the MB were obtained in all patients and from the SB in six patients. A per-strut analysis showed an uncovered strut rate of $0.7 \%$ and an incompletely-apposed strut rate of $0.8 \%$. Most incompletely-apposed struts were found at the bifurcation region, in the luminal half facing towards the SB. Mean NIH thickness in the proximal MB, distal $\mathrm{MB}$ and $\mathrm{SB}$ were $0.14 \pm 0.11,0.19 \pm 0.11$, and $0.34 \pm$
\end{abstract}

M. J. Grundeken · R. P. Kraak · P. Woudstra ·

T. G. van Leeuwen - K. T. Koch - J. G. Tijssen •

R. J. de Winter · J. J. Wykrzykowska (凹)

Department of Cardiology, The Heart Center, Academic Medical

Center, University of Amsterdam, B2-127, Meibergdreef 9,

1105 AZ Amsterdam, The Netherlands

e-mail: j.j.wykrzykowska@amc.uva.nl

M. J. Grundeken

e-mail: maikgrundeken@hotmail.com

H. M. Garcia-Garcia

The Thorax Center, Erasmus Medical Center, Erasmus

University Rotterdam, Rotterdam, The Netherlands

D. M. de Bruin

Department of Biomedical Engineering and Physics, Academic

Medical Center, University of Amsterdam, Amsterdam,

The Netherlands
$0.19 \mathrm{~mm}$, respectively, with a variety of growth patterns observed in the SB. We found good vascular healing of the DES in the MB, while healing was less favourably in the SB part. Furthermore, we observed a variety of NIH growth patterns in this SB part and more studies are needed to investigate the relation between growth patterns and clinical outcomes.

Keywords Coronary artery disease $\cdot$ Percutaneous coronary intervention - Coronary bifurcation lesion . Bifurcation lesion - Bifurcation stent - Tryton stent . Optical coherence tomography $\cdot$ Three-dimensional optical coherence tomography

\section{Introduction}

Coronary bifurcation lesions accounts for approximately $15 \%$ of all percutaneous coronary interventions (PCI) performed [1]. Percutaneous treatment of bifurcation lesions remains technically challenging and is associated with an increased risk of in-stent restenosis and stent thrombosis, when compared with non-bifurcation lesions [2, 3]. Therefore, several dedicated bifurcation devices, such as the Tryton side branch (SB) stent ${ }^{\mathrm{TM}}$ (Tryton Medical, Durham, NC, USA) have been developed to improve clinical outcomes after PCI of bifurcation lesions. The Tryton stent is a SB stent which is used in combination with a regular drug-eluting stent (DES) in the main branch (MB) and could be considered as a modified culotte technique [4]. Although being a bare-metal stent (BMS), previous observational studies have consistently shown low target lesion revascularization rates of $\sim 4 \%$ at 6-12 months of follow-up (FU) [5-9]. It is argued whether a drug-eluting version of this device would further improve 
clinical outcomes. Some argue that two DES in close proximity will increase stent thrombosis rates [10, 11], others are convinced drug-elution is essential to prevent excessive SB neo-intimal hyperplasia (NIH). Therefore, more insights in NIH growth patterns of the Tryton treatment strategy are needed. Optical coherence tomography (OCT) has a higher resolution compared to intravascular ultrasound ( $\sim 20$ vs $\sim 200$ micron) and is therefore suitable to perform a per-strut analysis evaluating strut apposition, strut coverage, and per-strut NIH growth. We present a 1-year FU study evaluating the NIH growth patterns of the Tryton strategy by using quantitative coronary angiography (QCA) and OCT analyses.

\section{Methods}

\section{Setting}

Our center, the Academic Medical Center-University of Amsterdam, is a large referral hospital with an annual PCI volume of $\sim 2,000$ procedures. All PCIs and adjunctive pharmacological treatment were performed according to the current guidelines.

\section{Device}

The Tryton stent has been extensively described elsewhere $[4,12]$. It is a balloon-expendable cobalt-chromium slotted-tube BMS. The stent is placed from the proximal MB into the SB using four radio-opaque markers, after which a regular DES is placed from the proximal to distal MB through the Tryton stent. The Tryton technique could be considered as a facilitated culotte technique. The stent design consists of three zones (distal SB zone, transition zone, and proximal $\mathrm{MB}$ zone) providing optimal scaffolding of the SB while minimizing the amount of metal in the MB. It is recommended to end the procedure with final kissing balloon inflation.

\section{Study design}

The study cohort consisted of ten patients in whom PCI of a bifurcation lesion was performed using the Tryton stent in our center between January 2011 and April 2012. Patients were asked to return for angiographic FU with OCT after 9 months. All patients who received a Tryton during the study period were eligible for inclusion except for patients who already underwent a re-PCI involving the target vessel within 9 months. Written informed consent was obtained from all patients prior to the repeat angiography. After repeat coronary angiography was performed, using the same views as before and after the initial procedure, OCT pullbacks were performed in both MB and SB. OCT pullbacks were not performed during the baseline procedure.

Quantitative coronary angiography

Coronary angiograms were analyzed offline using dedicated bifurcation software (QAngioXA version 7.3, Medis, Leiden, The Netherlands). Matched baseline, post-PCI and FU views were selected from the available angiograms. The bifurcation lesions were classified using the Medina classification [13]. All QCA analyses were performed in an end-diastolic frame, after calibration using the contrast-filled guiding catheter. Minimal lumen diameters (MLD) and lesion stenoses were obtained at baseline, post-procedure and during FU. Acute gain was calculated by subtracting the MLD at baseline from the MLD post procedure. Late luminal loss (LLL) was calculated by subtracting the MLD at FU from the MLD post procedure. QCA analyses were performed both in-stent and in-segment (in-stent plus $5 \mathrm{~mm}$ edges) for the following segments: proximal $\mathrm{MB}$, distal $\mathrm{MB}, \mathrm{SB}$, and for the ostial ( $<3 \mathrm{~mm}$ from the carina) SB and distal MB.

Optical coherence tomography

OCT pullbacks were obtained using the Ilumien frequencydomain system (St Jude Medical. St Paul, Minnesota, USA). OCT imaging catheters were advanced over a conventional, 0.014-inch guidewire and pullbacks were performed during continuous X-ray contrast injection of $4 \mathrm{ml} / \mathrm{s}$ at a maximum pressure of 300 psi using an injection pump. Images were acquired at 100 frames/s at a pullback speed of $20 \mathrm{~mm} / \mathrm{s}$. After calibration using the reflection of the imaging catheter, analyses of the acquired OCT images were performed offline using the C7 system (St Jude Medical. St Paul, Minnesota, USA). OCT analyses were performed separately in the following segments: distal MB, proximal MB, total SB (starting from the carina), ostial SB ( $<3 \mathrm{~mm}$ from the carina), distal SB ( $>3 \mathrm{~mm}$ from the carina), and the MB bifurcation region. The MB bifurcation region was further divided in a luminal half 'toward the SB' and a luminal half 'opposite the SB' (Fig. 1). Analyses were performed in each third frame $(0.6 \mathrm{~mm}$ interval) for the proximal MB, distal MB, and SB segments, whereas each frame $(0.2 \mathrm{~mm}$ interval) was analysed for the MB at the level of the SB segment. Luminal borders and stent contours were traced manually in each analysed frame. The NIH area was calculated by subtracting the lumen crosssectional area from the stent cross sectional area. The percent $\mathrm{NIH}$ was calculated by dividing the NIH area by the stent area, multiplied by 100 . To assess for asymmetric stent expansion, a stent eccentricity index was determined by dividing the minimal stent diameter by the maximal stent diameter in each frame, as previously described [14]. A per-strut analysis was performed in each analysed frame. Strut apposition was 
Fig. 1 Schematic drawing illustrating the different regions used for OCT analyses. At the main branch $(\mathrm{MB})$ bifurcation region, cross sections were analysed in two halves separately: a luminal half toward the side branch $(\mathrm{SB})$, and a luminal half opposite the SB

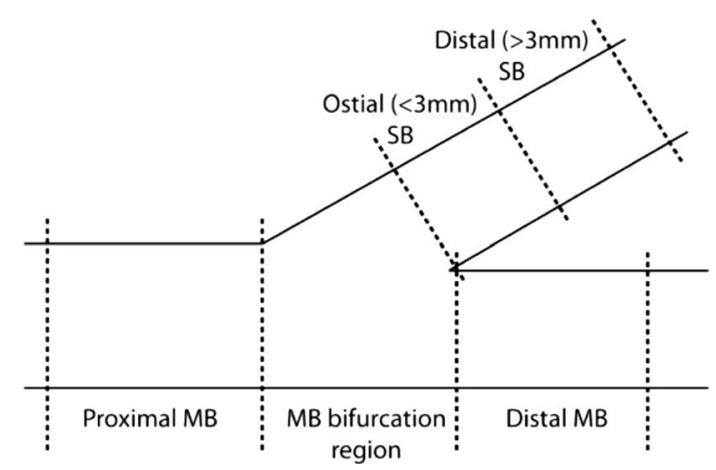

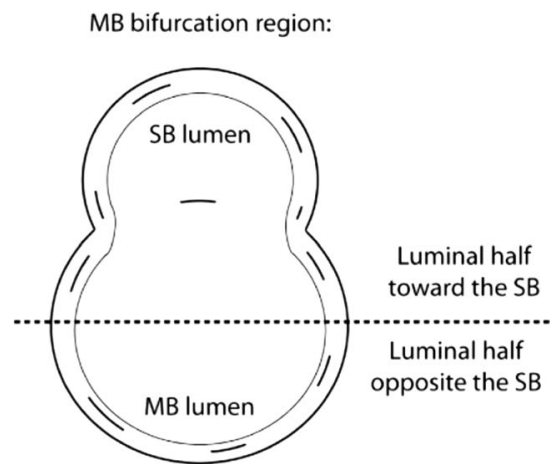

Table 1 Baseline patient and angiographic characteristics

\begin{tabular}{|c|c|}
\hline & $\mathrm{n}=10$ \\
\hline \multicolumn{2}{|l|}{ Baseline clinical characteristics } \\
\hline Age & $66( \pm 9)$ \\
\hline Male & $6(60 \%)$ \\
\hline Current smoker & $1(10 \%)$ \\
\hline Previous MI & $2(20 \%)$ \\
\hline Prior revascularization & $2(20 \%)$ \\
\hline Diabetes mellitus & $2(20 \%)$ \\
\hline Hypertension & $3(30 \%)$ \\
\hline Hypercholesterolemia & $5(50 \%)$ \\
\hline \multicolumn{2}{|l|}{ Indication for PCI } \\
\hline Stable angina & $6(60 \%)$ \\
\hline Unstable angina & $1(10 \%)$ \\
\hline NSTEMI & $2(20 \%)$ \\
\hline STEMI & $1(10 \%)$ \\
\hline \multicolumn{2}{|l|}{ Angiographic characteristics } \\
\hline \multicolumn{2}{|l|}{ Bifurcation location } \\
\hline Left main-RCx & $1(10 \%)$ \\
\hline LAD_diagonal branch & $8(80 \%)$ \\
\hline $\mathrm{RCA}-\mathrm{RDP}$ & $1(10 \%)$ \\
\hline \multicolumn{2}{|l|}{ Medina classification (on QCA) } \\
\hline $1,1,1$ & $1(10 \%)$ \\
\hline $1,0,1$ & $2(20 \%)$ \\
\hline $0,0,1$ & $2(20 \%)$ \\
\hline $1,1,0$ & $3(30 \%)$ \\
\hline $1,0,0$ & $1(10 \%)$ \\
\hline \multicolumn{2}{|l|}{ Lesion length (on QCA) } \\
\hline Proximal main branch (mm) & $6.5( \pm 3.3)$ \\
\hline Distal main branch (mm) & $6.0( \pm 4.7)$ \\
\hline Side branch $(\mathrm{mm})$ & $3.2( \pm 1.8)$ \\
\hline Overall TIMI 3 flow & $10(100 \%)$ \\
\hline
\end{tabular}

$M I$ myocardial infarction, $P C I$ percutaneous coronary intervention, NSTEMI non ST-segment elevation myocardial infarction, STEMI ST-segment elevation myocardial infarction, $R C x$ ramus circumflex artery, $L A D$ left anterior descending artery, $R C A$ right coronary artery, $R D P$ ramus descendens posterior, $Q C A$ quantitative coronary angiography, TIMI thrombolysis in myocardial infarction considered incomplete if the distance from the endoluminal stent surface to the vessel wall was greater than the thickness of the strut (metal plus polymer, if applicable) in the distal $\mathrm{MB}$, total $\mathrm{SB}$ and $\mathrm{MB}$ bifurcation region, whereas in the proximal MB, where Tryton and DES overlap, strut apposition was defined incomplete if the distance from the endoluminal cell surface to the vessel wall was greater than the strut thickness of Tryton plus DES used. Struts were classified as uncovered if any part of the strut was exposed to the lumen with no visible tissue coverage. NIH thickness was measured for every covered strut by measuring the distance from the strut to the luminal border.

To evaluate NIH growth patterns within the Tryton SB zone, mean per-strut NIH for each frame (in $\mathrm{mm}$ ) was plotted against the distance of the analysed frame from the carina (in mm). Furthermore, the stent eccentricity index was plotted against the distance from the carina (in $\mathrm{mm}$ ) to visually assess whether stent eccentricity influences growth patterns within the SB zone of the Tryton stent.

Three-dimensional optical coherence tomography reconstructions

Three-dimensional (3D) OCT images were reconstructed offline using volume-rendering software $\left(\right.$ AMIRA $^{\circledR}$ software, visualization science group, FEI company). SB ostia were visually assessed from the patients in whom nonapposed side branch (NASB) struts were seen during twodimensional OCT analyses. The 3D-OCT reconstructions were used to assess the position of the NASB struts relative to the SB ostium.

\section{Results}

Baseline patients and angiographic characteristics

Baseline patient and angiographic characteristics are shown in Table 1 . The majority ( $60 \%)$ of the patients were 
Table 2 Procedural characteristics

\begin{tabular}{lc}
\hline & $\mathrm{n}=10$ \\
\hline Variable & $8(80 \%)$ \\
MB predilatation & $9(90 \%)$ \\
SB predilatation & \\
Tryton used & $6(60 \%)$ \\
$3.0-2.5 \times 19 \mathrm{~mm}$ & $3(30 \%)$ \\
$3.5-2.5 \times 19 \mathrm{~mm}$ & $1(10 \%)$ \\
$3.5-3.0 \times 18 \mathrm{~mm}$ & \\
Main branch stent used & $9(90 \%)$ \\
Xience prime & $1(10 \%)$ \\
None & $3.0(3.0-3.5)$ \\
Diameter MB stent & $18(13.5-28)$ \\
Length MB stent & $4(40 \%)$ \\
Additional stenting in target vessel & $3(30 \%)$ \\
MB & $1(10 \%)$ \\
SB & $9(90 \%)$ \\
Final kissing balloon inflation & $10(100 \%)$ \\
Post procedural TIMI 3 flow &
\end{tabular}

$M B$ main branch, $S B$ side branch, TIMI thrombolysis in myocardial infarction

male with a mean age of 66 years. Indication for PCI included stable angina in $60 \%$, unstable angina in $10 \%$, non ST-segment elevation myocardial infarction (NSTE$\mathrm{MI}$ ) in $20 \%$, and ST-segment elevation myocardial infarction (STEMI) in the remaining $10 \%$. Most bifurcations were left anterior descending (LAD)—diagonal branch bifurcations. One left main bifurcation lesion was included in the current study.

Procedural characteristics and clinical outcomes

Procedural characteristics are summarized in Table 2. MB and SB pre-dilatations were performed in most cases (80 and $90 \%$, respectively). Xience (Abbott Vascular, Inc., Illinois, USA) everolimus-eluting DES was used as MB stent in $90 \%$ of the cases, while in the remaining case no MB stent was used. Final kissing balloon inflation was performed in $90 \%$ of the cases. In one case, SB wiring through the MB stent was unsuccessful after multiple attempts. None of the patients experienced any adverse event since the Tryton implantation up to the repeat angiography.

Quantitative coronary angiography

Mean time from PCI to FU angiography was $393 \pm 103$ days. QCA results are shown in Tables 3 and 4. In-stent acute gain was $1.78 \pm 0.94,1.18 \pm 0.45$, and $0.81 \pm 0.42 \mathrm{~mm}$ in the proximal $\mathrm{MB}$, distal $\mathrm{MB}$ and $\mathrm{SB}$, respectively, whereas in-stent acute gain was $1.14 \pm 0.56$ for the ostial distal MB and $0.93 \pm 0.58$ for the ostial SB. In-stent LLL in the proximal MB, distal MB, and $\mathrm{SB}$ was $0.27 \pm 0.31,0.24 \pm 0.28$, and $0.49 \pm 0.32 \mathrm{~mm}$, respectively (Fig. 2). Mean distal bifurcation angles were $54 \pm 17,51 \pm 19$, and $54 \pm 16$ degrees at baseline, postPCI and FU, respectively. Binary restenosis was found in only one patient (SB stenosis of $57 \%$ during FU). This patient was asymptomatic and had no angina during repeat angiography. No repeat intervention was performed.

Optical coherence tomography

OCT pullbacks in the MB were obtained in all cases, whereas OCT pullbacks in the SB could not be obtained in 4 cases due to kinking of the OCT catheter while attempting to advance into the SB. OCT analyses are summarized in Table 5.

\section{Per strut analysis}

In total, 6,127 struts were analysed in 515 frames. Only $0.7 \%$ of total struts were uncovered, while $0.8 \%$ of the struts were incompletely apposed. The region with the most incompletely apposed struts was the luminal half facing toward the $\mathrm{SB}$ of the MB bifurcation region, with $6.2 \%$ of the struts being incompletely apposed in that region. Sixteen percent of these struts were also uncovered with tissue (Table 5).

\section{Neointimal thickness}

Average per-strut NIH thickness in the proximal MB, distal $\mathrm{MB}$ and SB were $0.14 \pm 0.11,0.19 \pm 0.11$, and $0.34 \pm 0.19 \mathrm{~mm}$, respectively. $\mathrm{NIH}$ thickness in the $\mathrm{MB}$ bifurcation region was comparable with the proximal en distal MB segments $(0.20 \pm 0.14$ opposite to the $\mathrm{SB}$ and $0.16 \pm 0.13 \mathrm{~mm}$ towards the $\mathrm{SB}$, respectively).

\section{Patterns of neoitimal thickness in relation to the $S B$ ostium in individual patients}

We observed differences between ostial and distal mean NIH thickness in individual patients (Fig. 3). In two patients, NIH thickness was smaller in the ostium of the SB as compared with the distal SB (B and E), in two other patients, there was no difference $(C$ and $D)$, whereas in the remaining two patients $\mathrm{NIH}$ thickness was larger in the ostium when compared with the distal SB (A and F). In Fig. 4, the stent eccentricity index on the vertical axis was plotted against the distances from the carina on the horizontal axis showing stent eccentricity of the Tryton stent at 
Table 3 Pre- and post-procedural quantitative coronary angiography

\begin{tabular}{|c|c|c|c|c|c|c|c|c|}
\hline & \multicolumn{3}{|l|}{ Pre-procedure } & \multicolumn{5}{|l|}{ Post-procedure } \\
\hline & $\operatorname{MLD}(\mathrm{mm})^{*}$ & RVD (mm) & DS (\%) & $\operatorname{MLD}(\mathrm{mm})^{\dagger}$ & RVD (mm) & DS $(\%)$ & Acute gain $(\mathrm{mm}) *$ & $P$ value \\
\hline \multicolumn{9}{|l|}{ In-stent } \\
\hline Proximal MB & $1.15( \pm 0.67)$ & $3.99( \pm 0.60)$ & $69( \pm 18)$ & $3.09( \pm 0.60)$ & $3.85( \pm 0.59)$ & $15( \pm 10)$ & $1.78( \pm 0.94)$ & 0.0005 \\
\hline Distal MB & $1.21( \pm 0.60)$ & $2.48( \pm 0.44)$ & $51( \pm 21)$ & $2.43( \pm 0.42)$ & $2.63( \pm 0.59)$ & $6( \pm 12)$ & $1.18( \pm 0.45)$ & 0.0002 \\
\hline SB & $1.03( \pm 0.54)$ & $2.15( \pm 0.27)$ & $52( \pm 22)$ & $1.92( \pm 0.30)$ & $2.32( \pm 0.30)$ & $16( \pm 9)$ & $0.81( \pm 0.42)$ & 0.0004 \\
\hline Ostium distal MB & $1.50( \pm 0.64)$ & $2.53( \pm 0.43)$ & $40( \pm 25)$ & $2.75( \pm 0.46)$ & $2.74( \pm 0.29)$ & $-0.5( \pm 13)$ & $1.14( \pm 0.56)$ & 0.0007 \\
\hline Ostium SB & $1.10( \pm 0.67)$ & $2.18( \pm 0.29)$ & $50( \pm 25)$ & $2.09( \pm 0.33)$ & $2.38( \pm 0.28)$ & $12( \pm 13)$ & $0.93( \pm 0.58)$ & 0.0014 \\
\hline \multicolumn{9}{|l|}{ In-segment } \\
\hline Proximal MB & $1.15( \pm 0.67)$ & $3.99( \pm 0.60)$ & $69( \pm 18)$ & $2.90( \pm 0.70)$ & $3.76( \pm 0.71)$ & $17( \pm 11)$ & $1.57( \pm 1.08)$ & 0.0024 \\
\hline Distal MB & $1.16( \pm 0.48)$ & $2.43( \pm 0.40)$ & $51( \pm 20)$ & $1.89( \pm 0.20)$ & $2.43( \pm 0.21)$ & $21( \pm 11)$ & $0.76( \pm 0.60)$ & 0.0095 \\
\hline SB & $1.00( \pm 0.52)$ & $2.11( \pm 0.34)$ & $52( \pm 22)$ & $1.72( \pm 0.43)$ & $2.17( \pm 0.36)$ & $20( \pm 11)$ & $0.64( \pm 0.44)$ & 0.0023 \\
\hline
\end{tabular}

$M B$ main branch, $S B$ side branch, $M L D$ minimal lumen diameter, $D S$ diameter stenosis

* Measurements/calculations based on 9 angiographies (from 1 patient, the software was unable to trace luminal borders and applying the bifurcation algorithm correctly)

$\dagger$ Mean MLD based on 10 angiographies
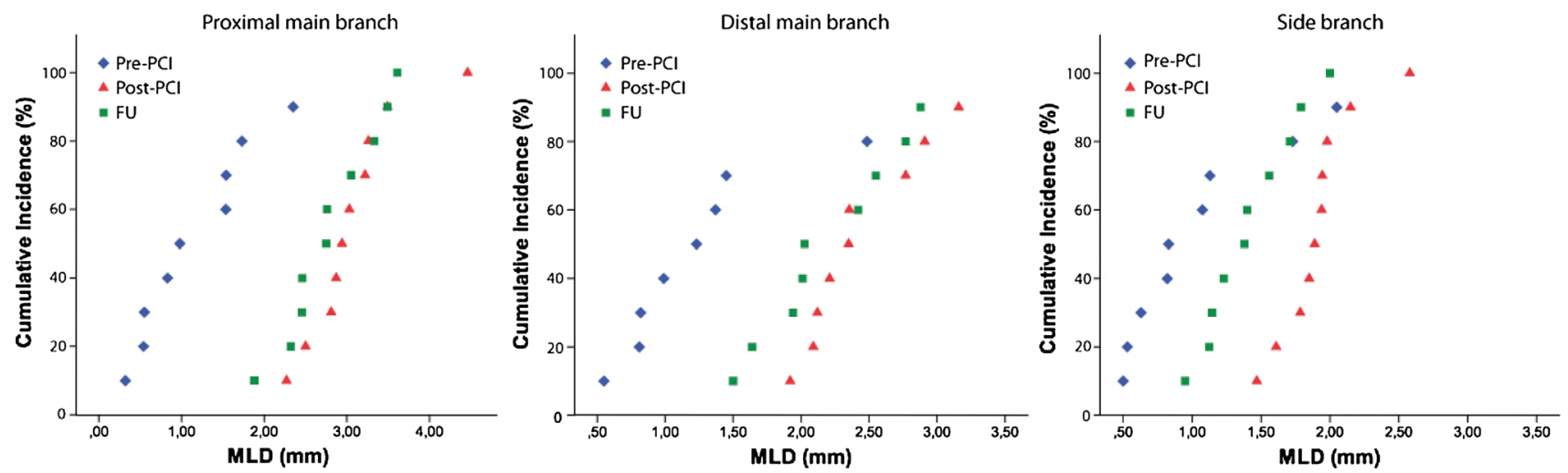

Fig. 2 Cumulative incidence curves of minimal lumen diameters (MLD) of the proximal main branch, distal main branch, and side branch

Table 4 FU quantitative coronary angiography

$M B$ main branch, $S B$ side branch, $M L D$ minimal lumen diameter, $D S$ diameter stenosis

\begin{tabular}{lccccc}
\hline & MLD $(\mathrm{mm})$ & RVD $(\mathrm{mm})$ & DS $(\%)$ & Late luminal loss $(\mathrm{mm})$ & $P$ value \\
\hline In-stent & & & & & \\
$\quad$ Proximal MB & $2.81( \pm 0.56)$ & $4.42( \pm 0.70)$ & $21( \pm 11)$ & $0.27( \pm 0.31)$ & 0.0022 \\
Distal MB & $2.19( \pm 0.49)$ & $2.57( \pm 0.31)$ & $15( \pm 12)$ & $0.24( \pm 0.28)$ & 0.0345 \\
SB & $1.43( \pm 0.33)$ & $2.15( \pm 0.26)$ & $33( \pm 14)$ & $0.49( \pm 0.32)$ & 0.0009 \\
Ostium distal MB & $2.47( \pm 0.53)$ & $2.70( \pm 0.30)$ & $9( \pm 15)$ & $0.27( \pm 0.26)$ & 0.0131 \\
Ostium SB & $1.51( \pm 0.38)$ & $2.17( \pm 0.25)$ & $30( \pm 15)$ & $0.58( \pm 0.38)$ & 0.0010 \\
In-segment & & & & & \\
Proximal MB & $2.73( \pm 0.57)$ & $4.38( \pm 0.79)$ & $23( \pm 11)$ & $0.18( \pm 0.34)$ & 0.1335 \\
Distal MB & $2.00( \pm 0.37)$ & $2.46( \pm 0.24)$ & $18( \pm 11)$ & $-0.10( \pm 0.41)$ & 0.4833 \\
SB & $1.42( \pm 0.32)$ & $2.14( \pm 0.27)$ & $33( \pm 14)$ & $0.30( \pm 0.37)$ & 0.0307 \\
\hline
\end{tabular}

the SB ostium in some cases (B and E). In Fig. 5, the stent eccentricity index of the most ostial SB frame (on the vertical axis) was plotted against the ratios of NIH thickness of the ostial versus distal SB (on the horizontal axis) showing that ostial stent eccentricity is associated with NIH growth being more pronounced distally in the SB. 
Table 5 One-year OCT analyses $(n=10)$

\begin{tabular}{|c|c|c|c|c|c|c|}
\hline & Total & Proximal MB & Distal MB & SB* & $\begin{array}{l}\text { Bifurcation } \\
\text { area: opposite SB }\end{array}$ & $\begin{array}{l}\text { Bifurcation } \\
\text { area: toward SB }\end{array}$ \\
\hline Per frame analysis: & $(\mathrm{n}=515)$ & $(\mathrm{n}=187)$ & $(\mathrm{n}=257)$ & $(\mathrm{n}=71)$ & & \\
\hline Stent area $\left(\mathrm{mm}^{2}\right)$ & $7.84( \pm 2.26)$ & $9.56( \pm 2.06)$ & $7.43( \pm 1.47)$ & $4.75( \pm 0.74)$ & & \\
\hline Lumen area $\left(\mathrm{mm}^{2}\right)$ & $6.24( \pm 2.52)$ & $8.15( \pm 2.42)$ & $5.86( \pm 1.35)$ & $2.56( \pm 0.73)$ & & \\
\hline $\mathrm{NIH}$ area $\left(\mathrm{mm}^{2}\right)$ & $1.57( \pm 0.89)$ & $1.36( \pm 0.98)$ & $1.54( \pm 0.72)$ & $2.23( \pm 0.89)$ & & \\
\hline Percent NIH area $(\%)$ & $0.22( \pm 0.15)$ & $0.15( \pm 0.12)$ & $0.21( \pm 0.09)$ & $0.46( \pm 0.16)$ & & \\
\hline Per strut analysis: & $(\mathrm{n}=6127)$ & $(\mathrm{n}=2155)$ & $(\mathrm{n}=2495)$ & $(\mathrm{n}=534)$ & $(n=424)$ & $(\mathrm{n}=519)$ \\
\hline NIH thickness (mm) & $0.18( \pm 0.13)$ & $0.14( \pm 0.11)$ & $0.19( \pm 0.11)$ & $0.34( \pm 0.19)$ & $0.20( \pm 0.14)$ & $0.16( \pm 0.13)$ \\
\hline Uncovered struts (\%) & $41(0.7)$ & $8(0.4)$ & $3(0.1)$ & $0(-)$ & $16(3.8)$ & $14(2.7)$ \\
\hline Incomplete apposed struts (\%) & $50(0.8)$ & $12(0.6)$ & $6(0.2)$ & $0(-)$ & $0(-)$ & $32(6.2)$ \\
\hline $\begin{array}{l}\text { Percentage uncovered from } \\
\text { incompletely apposed struts }(\%)\end{array}$ & $5(10)$ & $0(-)$ & $0(-)$ & NA & NA & $5(16)$ \\
\hline
\end{tabular}

$M B$ main branch, $S B$ side branch, $N I H$ neo-intimal hyperplasia, $N A$ not applicable

* Based on 6 pullbacks

Three-dimensional optical coherence tomography

Three-dimensional OCT images from the patients in whom NASB struts were seen during 2D-OCT analyses are shown in Fig. 6. While viewing at the SB ostia from outside the vessel and from an endoluminal point-of-view downstream, there were no stent struts clearly visualized which obstructed the SB ostium. After rotating the 3Drecontruction in such way that the NASB struts are best visible, which was a perpendicular view at the SB ostium in all cases, it could be clearly demonstrated that the NASB struts are situated at the distal end of the SB ostia, at the (neo-) carina. In two patients, these NASB struts separated the SB ostia in two separate compartments, while in the other 4 patients only a single compartment was seen.

\section{Discussion}

Our study provides detailed information on neo-intimal growth patterns of the Tryton bifurcation treatment. Overall, QCA and OCT analyses demonstrated good healing of the proximal and distal $\mathrm{MB}$ with an in-stent LLL of $0.27 \pm 0.31$ and $0.24 \pm 0.28 \mathrm{~mm}$, respectively and NIH thickness during FU of $0.14 \pm 0.11$ and $0.19 \pm 0.11 \mathrm{~mm}$, respectively, while healing of the SB is less favourable with a LLL of $0.49 \pm 0.32 \mathrm{~mm}$ and a mean NIH thickness of $0.34 \pm 0.19 \mathrm{~mm}$. Furthermore, 3D-OCT reconstructions of the SB ostia of patients with jailed SB struts demonstrated that the jailed SB struts were situated only at the distal end of the ostia, at the carinal level.
Previous angiographic follow-up studies

Up-to-date, two previous studies with angiographic FU have been performed with the Tryton SB stent. While the Tryton first-in-man study showed in-stent LLL of $0.25,0.0$, and $0.17 \mathrm{~mm}$ in the proximal $\mathrm{MB}$, distal $\mathrm{MB}$, and $\mathrm{SB}$ respectively [5], the PYTON study demonstrated contrasting results, showing in-stent LLL of 0.34, 0.29, and $0.57 \mathrm{~mm}$, respectively [15]. Our findings are comparable with this latter study, showing in-stent LLL of $0.27,0.24$, and $0.49 \mathrm{~mm}$, respectively. Although these findings might be interpreted as unfavourable compared with historical data from clinical trials on the Xience stent showing instent LLL of $0.10-0.19 \mathrm{~mm}$ [16-19], they are not dissimilar to QCA results of a substudy in complex lesions, including bifurcation lesions, from one of the large trials on Xience DES showing a LLL of $0.23 \pm 0.44 \mathrm{~mm}$ [20]. Although the largest LLL was seen in the bare-metal Tryton SB part, the LLL of $0.49 \mathrm{~mm}$ in fact compares very well with historical data on straight, tubular BMS showing LLL of 0.70-1.00 [21-24]. The relative low LLL we found in the current study might be explained by the fact that only $\sim 7 \mathrm{~mm}$ of the Tryton stent is positioned in the SB, while the occurrence of BMS in-stent restenosis is inversely related to lesion length [25].

Previous optical coherence tomography follow-up studies

Until now, the above-mentioned PYTON study was the only study published to-date, which evaluated the Tryton treatment with intravascular imaging during FU. The investigators of this study used OCT to assess strut 

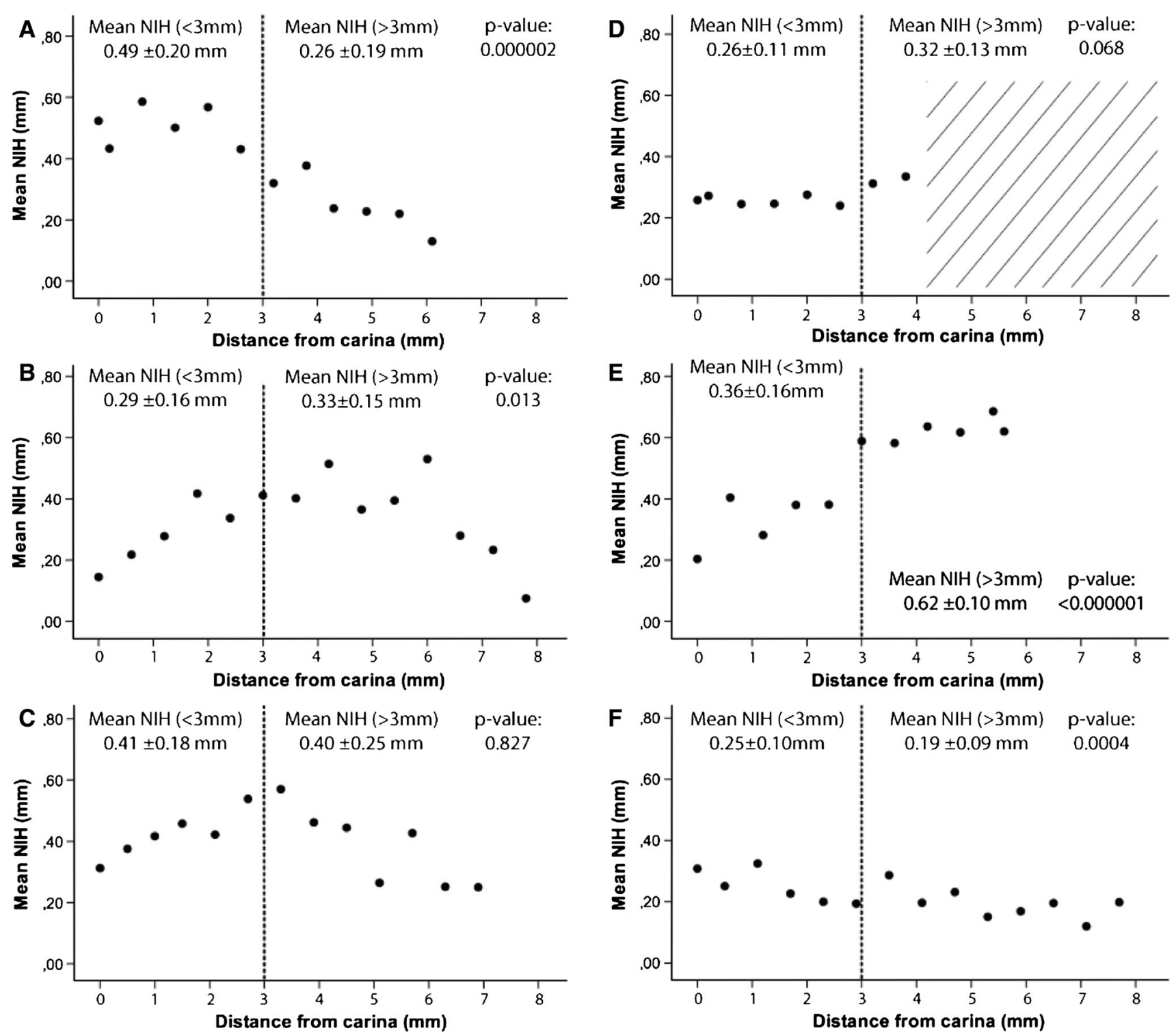

Fig. 3 Mean neo-intimal hyperplasia (NIH) thickness per analysed frame (vertical axis), plotted against the distance of the frame from the carina (horizontal axis). Each letter a-f indicating an individual patient. The dotted line indicates a $3 \mathrm{~mm}$ distance from the carina. $P$ values are between mean NIH of the first $3 \mathrm{~mm}$ versus mean NIH

beyond $3 \mathrm{~mm}$ from the carina. The lines in panel $D$ illustrating missing values due to OCT images of insufficient quality, whereas in the other patients $(\mathbf{a}-\mathbf{c} ; \mathbf{e}-\mathbf{f})$, the whole Tryton could be visualized with OCT

apposition and coverage for the different segments. Our findings were similar to their findings in that most uncovered and incompletely apposed struts were at the bifurcation region, whereas none of the struts in the SB were unapposed or uncovered. While the PYTON investigators did not distinguish between struts opposing the SB ostium and struts facing the SB ostium, we found that all of the unapposed struts in the MB bifurcation region were located facing towards the SB. Interestingly, no incompletely apposed struts were observed opposite from the SB ostium, which has been shown with culotte stenting using

conventional DES after final kissing balloon inflation (the so-called 'napkin ring' effect) [26, 27]. It could be hypothesized that the open cell design of the Tryton stent prevents this napkin ring effect since the large cell size of Tryton in the MB zone does not limit MB DES expansion. On contrary to PYTON, we also performed a per-strut analysis regarding NIH thickness. The latter analysis has not been performed by PYTON investigators and in that respect it is a novel finding. We demonstrated a $\mathrm{NIH}$ thickness of 0.14 and $0.19 \mathrm{~mm}$ in the proximal and distal MB. These results compare well with previous OCT 

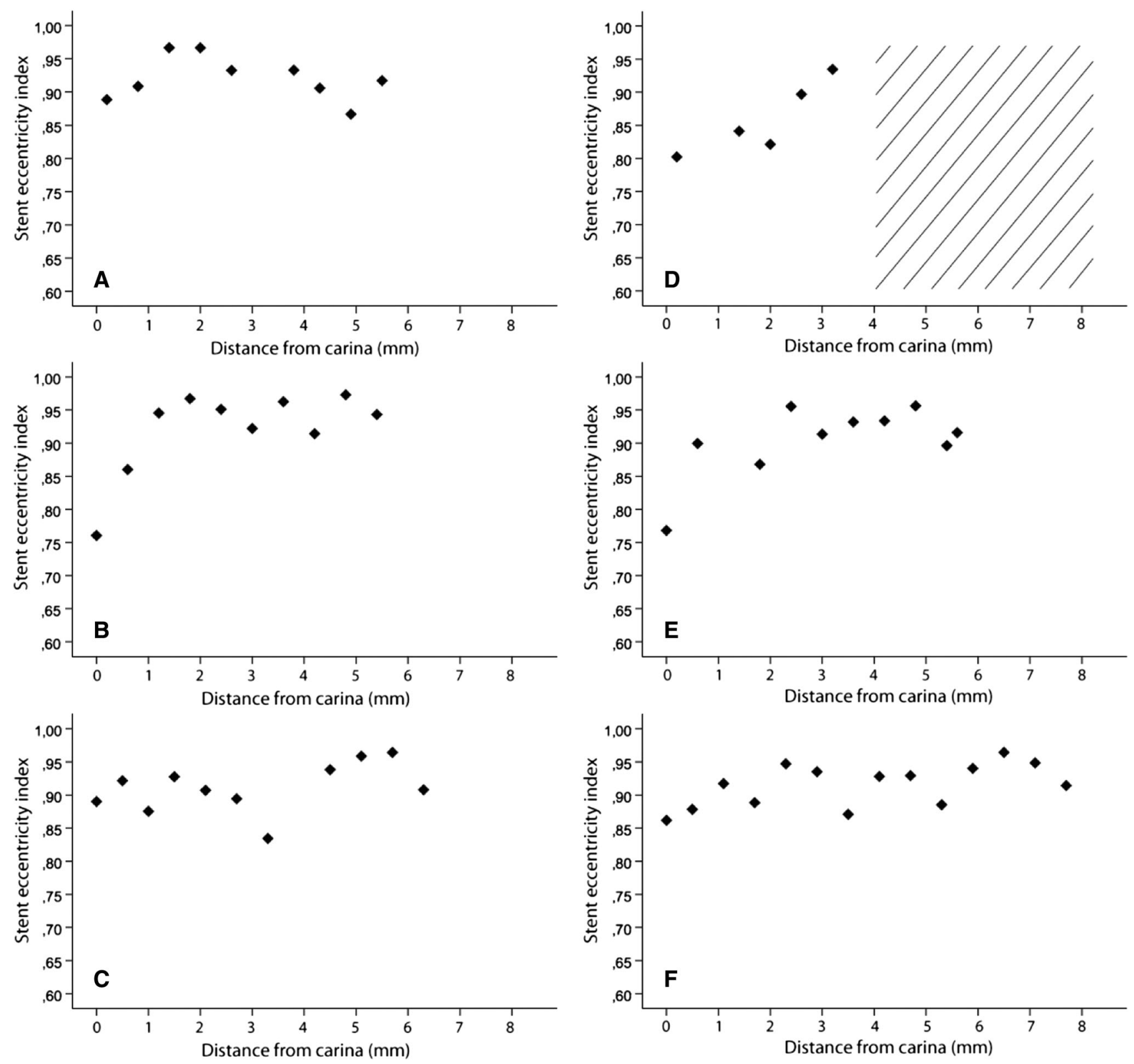

Fig. 4 Stent eccentricity index (vertical axis), plotted against the distance of the frame from the carina (horizontal axis). Each letter af indicating an individual patient. A stent eccentricity index of 1 corresponds to a perfect round stent shape, whereas a ratio below 1

suggests an eccentric stent shape. The lines in panel $D$ illustrating missing values due to OCT images of insufficient quality, whereas in the other patients $(\mathbf{a}-\mathbf{c} ; \mathbf{e}-\mathbf{f})$, the whole Tryton could be visualized with OCT

studies on the Xience stent in non-bifurcation studies, which showed a NIH thickness of $0.10-0.18 \mathrm{~mm}$ [28-31]. Furthermore, the SB NIH of $0.34 \mathrm{~mm}$ we found resembles the historical data evaluating BMS with OCT which consistently showed NIH thickness of $\sim 0.35 \mathrm{~mm}[32,33]$.

Neo-intimal growth patterns of the side branch

We explored the NIH growth patterns of the bare-metal SB by plotting the mean NIH of each frame against the distance

from the carina (Fig. 3). It seems that there is some variation in NIH growing patterns of the SB, although we have to be cautious to draw general conclusions based on the relative small sample size. We hypothesized that stent eccentricity at the SB ostium might influence the NIH growth more distally by disturbing flow patterns at the SB ostium. Indeed, we found that in the patients with more pronounced distal NIH growth, the most ostial stent contour was eccentric compared with the other growth patterns (Fig. 4), These findings need to be confirmed in a larger patient population. 


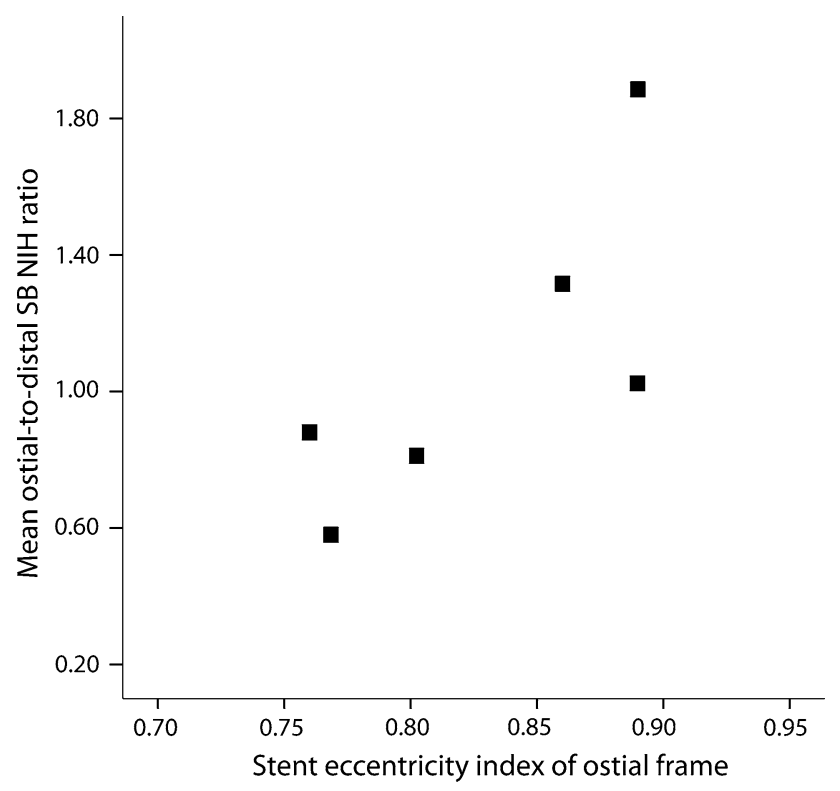

Fig. 5 Relation between the stent eccentricity index of the first side branch (SB) frame (at the level of the carina) on the vertical axis and the ratio of ostial $(<3 \mathrm{~mm}$ from carina) versus distal $(>3 \mathrm{~mm}$ from carina) mean neo-intimal hyperplasia (NIH) thickness in the SB (in $\mathrm{mm}$ ) on the horizontal axis. An ostial-to-distal SB NIH ratio $<1$ indicates more NIH growth distally, whereas an ostial-to-distal SB $\mathrm{NIH}$ ratio $>1$ indicates more NIH growth in the ostium

Interestingly, the patient with the most pronounced ostial NIH growth (patient "A" in Figs. 3 and 4), was the one patient who did not receive a stent in the MB, suggesting that drug from the MB stent does also influences NIH growth in the ostial SB part of Tryton.

Non-apposed side branch struts

As mentioned above, most of the incompletely apposed struts were found at the bifurcation region in the luminal half facing toward the SB. Most of these struts are NASB struts, jailing the SB on 2D-OCT. We performed 3D-OCT reconstructions to assess where these NASB struts are located relative to the $\mathrm{SB}$ ostium. We found that all of these NASB struts are located at the distal end of the SB ostium (at the carina), whereas none of the unapposed struts toward the SB could be visualized at the proximal side of the SB ostium, both with conventional 2D-OCT analyses as well as with visual assessment of 3D-OCT reconstructions. With 3D-OCT, we could visualize the shape of the NASB struts. When placed correctly, the transition zone of the Tryton stent should be positioned at the level of the carina. This zone consists of three panels connecting the undulating fronds in the MB with the zig-zag hoops more distally in the SB. Visual assessment of the shape of the NASB struts showing the typical zig-zag shaped appearance of the Xience hoops which implies that these NASB struts belong to the Xience DES in the MB rather than the
Tryton stent. Indeed, no NASB struts were found in the one patient who did not receive a MB stent. This suggests NASB struts in the Tryton procedure could be attributed to the MB stent and that re-crossing the most distal cell before final kissing balloon inflation is also vital in Tryton procedures as it is in other bifurcation treatment strategies [34-36], in order to minimize the amount of NASB struts which are associated with delayed healing [37].

Drug-coating the Tryton stent, is it a useful future development?

Our data showed that NIH growth within the SB is as could be expected from a BMS, showing equal results compared with historical QCA and OCT data on straight tubular BMS. It is therefore an attractive concept whether clinical outcomes could potentially improve further by drug-coating the bare-metal Tryton device, since drug-elution of conventional tubular stents has shown to improve clinical efficacy, especially with regard to in-stent restenosis [21]. Hypothesis-generating findings on the $\mathrm{NIH}$ distribution from the current study might be helpful in the decision if and where precisely to coat the Tryton stent with a drug in the future. First, our data showed that in patients receiving a DES in the MB, NIH growth in the SB ostium $(<3 \mathrm{~mm}$ from the carina) was quite modest, ranging from 0.25 to $0.41 \mathrm{~mm}$. NIH thickness was more pronounced in the distal $\mathrm{SB}$, ranging from 0.19 up to as high as $0.61 \mathrm{~mm}$. Second, none of the struts in the SB were incompletely apposed or uncovered. This may explain the excellent safety profile of the stent with low ST rates, as observed in previous observational studies [5-9]. Third, we found that all nonapposed struts in the bifurcation region were located toward the SB and with 3D-OCT we observed that all of these struts were located at the carina. Of these struts, $16 \%$ were uncovered, which confirms the findings of a previous autopsy study demonstrating impaired healing at the carina with DES [10]. It is likely that two DES in close proximity at the carina would impair healing even more, resulting in an increased risk of stent thrombosis, as shown in a recent meta-analysis comparing one with two DES used in bifurcation treatment [11]. In conclusion, if it were to be decided that Tryton will be coated with a drug, one might argue only to coat the distal SB zone rather than the whole stent, including the transition zone, to avoid a potential increased risk of ST due to two DES in close proximity at the carina impairing healing at the flow divider where flow is the highest, and thereby slowing the healing process.

Study limitations

We investigated a relative small patient population. However, this study provides detailed per-strut information on 


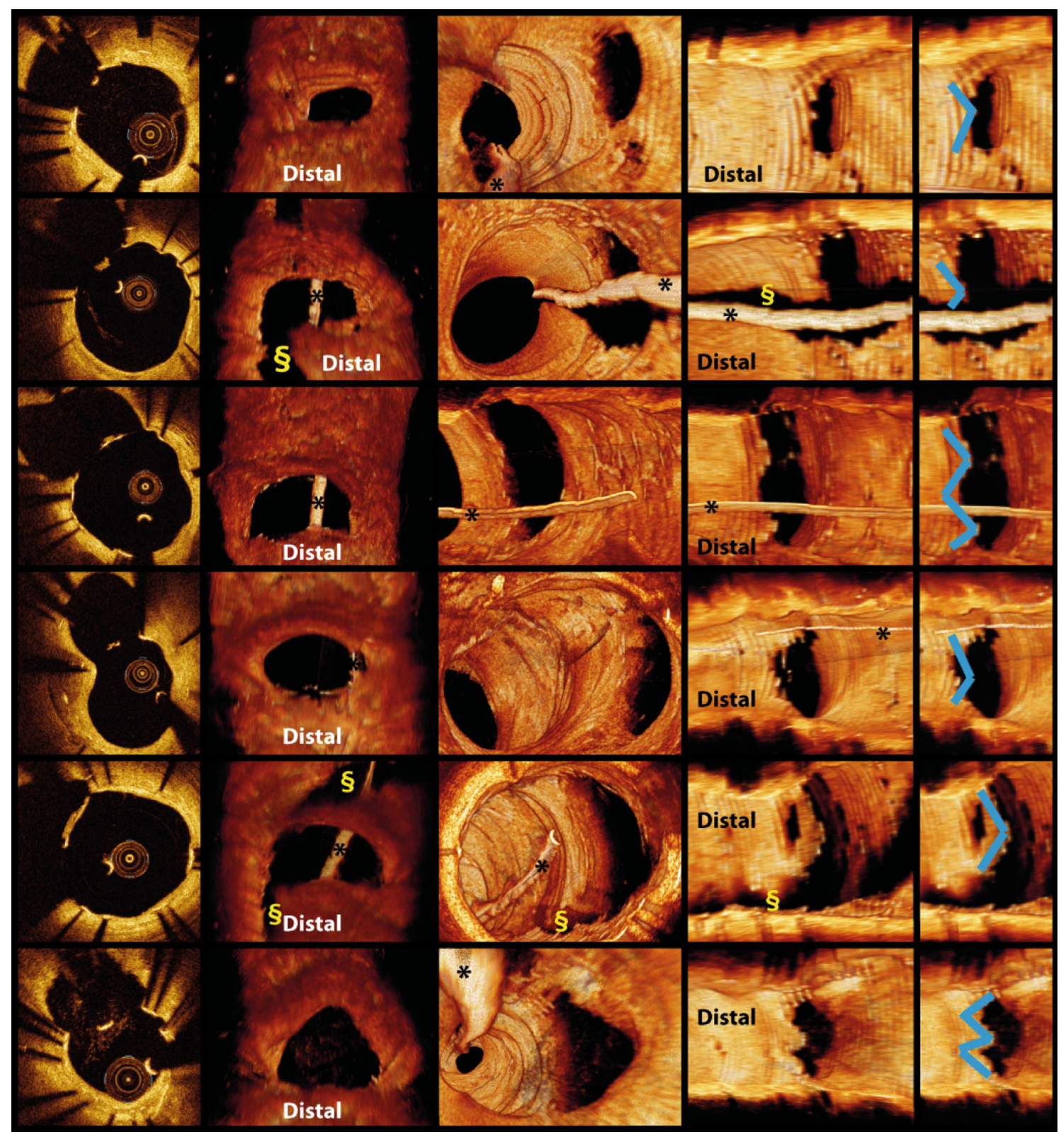

Fig. 6 Three-dimensional optical coherence tomography (3D-OCT) reconstructions illustrating the position of incompletely apposed struts relative to the side branch ostium. Panels horizontal from each other correspond with the same patient. In the most left panels, OCT still frames illustrating the incompletely apposed struts in the bifurcation region facing toward the side branch. The second left panels showing ostial views from outside the vessel. The third left panels showing 'fly-through' views looking downstream at the carina. In these

healing responses of the Tryton treatment. Nevertheless, due to the small sample size generalizability is limited and the current findings should be interpreted with caution and appreciated as hypothesis-generating. Initial Tryton implantation was performed according to the operator's discretion and patients who already received a repeat PCI of the Tryton-treated bifurcation lesion before 9 months were excluded for the current study, and thus a selection particular views, no struts were visualized which clearly obstructing the side branch ostium. The second right frames showing the views with the most optimal visualization of the non-apposed struts toward the side branch, which was best visualized perpendicular to the side branch ostium in all cases. The blue lines in the most right frames illustrating the shape of the non-apposed struts, which were typical 'zig-zag' hoops in all cases. (*) illustrates guidewires, whereas $(\S)$ illustrates guide wire shadows

bias has been introduced. The current findings should be appreciated as hypothesis-generating and its relation with clinical outcomes should be investigated in large clinical trials. Furthermore, we were not able to advance an OCT catheter in the SB in 4 from the 10 patients, which might introduce a selection bias for the OCT SB analyses. Finally, baseline (both pre-procedural and post-procedural) OCT imaging was not performed, which would have given 
more insights in technical aspects during placement and its relation to vascular healing at FU.

\section{Conclusions}

We found good vascular healing of the distal and proximal part of the MB DES. Healing of the bare-metal Tryton in the SB was less favourable compared to the MB DES, although at least comparable with historical data on healing of tubular BMS stents. The late loss of $0.49 \mathrm{~mm}$ in the SB limits the systematic use of Tryton in all bifurcations, especially in smaller side branches. Further, we found that most incompletely apposed and uncovered struts are located in the bifurcation region facing towards the SB ostium, and that these struts are located distal (near the carina) rather than proximal relative to the SB ostium.

Conflict of interest Maik Grundeken and Joanna Wykrzykowska receive consultancy fees from Tryton Medical. None of the other authors declared any relevant conflict of interest.

\section{References}

1. Sarno G, Lagerqvist B, Frobert O, Nilsson J, Olivecrona G, Omerovic E et al (2012) Lower risk of stent thrombosis and restenosis with unrestricted use of 'new-generation' drug-eluting stents: a report from the nationwide Swedish coronary angiography and angioplasty registry (SCAAR). Eur Heart J 33(5):606-613

2. Rathore S, Terashima M, Katoh O, Matsuo H, Tanaka N, Kinoshita Y et al (2009) Predictors of angiographic restenosis after drug eluting stents in the coronary arteries: contemporary practice in real world patients. EuroIntervention 5(3):349-354

3. van Werkum JW, Heestermans AA, Zomer AC, Kelder JC, Suttorp MJ, Rensing BJ et al (2009) Predictors of coronary stent thrombosis: the Dutch Stent Thrombosis Registry. J Am Coll Cardiol 53(16):1399-1409

4. Kaplan AV, Davis HR (2006) Tryton side-branch stent. EuroIntervention 2(2):270-271

5. Onuma Y, Muller R, Ramcharitar S, van Geuns RJ, Louvard Y, Morel MA et al (2008) Tryton I, First-In-Man (FIM) study: six month clinical and angiographic outcome, analysis with new quantitative coronary angiography dedicated for bifurcation lesions. EuroIntervention 3(5):546-552

6. Magro M, Wykrzykowska J, Serruys PW, Simsek C, Nauta S, Lesiak M et al (2011) Six-month clinical follow-up of the Tryton side branch stent for the treatment of bifurcation lesions: a two center registry analysis. Catheter Cardiovasc Interv 77(6):798-806

7. Agostoni P, Foley D, Lesiak M, Belkacemi A, Dens J, Kumsars I et al (2012) A prospective multicentre registry, evaluating realworld usage of the Tryton side branch stent: results of the E-Tryton 150/Benelux registry. EuroIntervention 7(11):1293-1300

8. Grundeken MJ, Smits M, Harskamp RE, Damman P, Woudstra P, Hoorweg AJ et al (2012) Six-month clinical outcomes of the Tryton Side Branch Stent for the treatment of bifurcation lesions. Neth Heart J 20(11):439-446

9. Grundeken MJ, Asgedom S, Damman P, Lesiak M, Norell MS, Garcia E et al (2013) Six-month and one-year clinical outcomes after placement of a dedicated coronary bifurcation stent: a patient-level pooled analysis of eight registry studies. EuroIntervention 9(2): 195-203

10. Nakazawa G, Yazdani SK, Finn AV, Vorpahl M, Kolodgie FD, Virmani R (2010) Pathological findings at bifurcation lesions: the impact of flow distribution on atherosclerosis and arterial healing after stent implantation. J Am Coll Cardiol 55(16):1679-1687

11. Zimarino M, Corazzini A, Ricci F, Di NM, De CR (2013) Late thrombosis after double versus single drug-eluting stent in the treatment of coronary bifurcations: a meta-analysis of randomized and observational studies. JACC Cardiovasc Interv 6(7):687-695

12. Magro M, van Geuns RJ (2010) TheTryton side branch stent. EuroIntervention 6(Suppl J):J147-J150

13. Medina A, de Suarez LJ, Pan M (2006) A new classification of coronary bifurcation lesions. Rev Esp Cardiol 59(2):183

14. Otake H, Shite J, Ako J, Shinke T, Tanino Y, Ogasawara D et al (2009) Local determinants of thrombus formation following sirolimus-eluting stent implantation assessed by optical coherence tomography. JACC Cardiovasc Interv 2(5):459-466

15. Dubois C, Adriaenssens T, Ughi G, Wiyono IS, Bennett J, Coosemans $M$ et al (2012) Healing responses after bifurcation stenting with the dedicated TRYTON side-branch Stent (TM) in combination with XIENCE-V (TM) stents: a clinical, angiography, fractional flow reserve and optical coherence tomography study. Catheter Cardiovasc Interv 81(3):E155-E164

16. Serruys PW, Ong AT, Piek JJ, Neumann FJ, van der Giessen WJ, Wiemer $\mathrm{M}$ et al (2005) A randomized comparison of a durable polymer everolimus-eluting stent with a bare metal coronary stent: the SPIRIT first trial. EuroIntervention 1(1):58-65

17. Serruys PW, Ruygrok P, Neuzner J, Piek JJ, Seth A, Schofer JJ et al (2006) A randomised comparison of an everolimus-eluting coronary stent with a paclitaxel-eluting coronary stent: the SPIRIT II trial. EuroIntervention 2(3):286-294

18. Stone GW, Midei M, Newman W, Sanz M, Hermiller JB, Williams J et al (2008) Comparison of an everolimus-eluting stent and a paclitaxel-eluting stent in patients with coronary artery disease: a randomized trial. JAMA 299(16):1903-1913

19. Kozuma K, Kimura T, Kadota K, Suwa S, Kimura K, Iwabuchi M et al (2013) Angiographic findings of everolimus-eluting as compared to sirolimus-eluting stents: angiographic sub-study from the Randomized Evaluation of sirolimus-eluting versus everolimus-eluting stent Trial (RESET). Cardiovasc Interv Ther 28(4):344-351

20. Stefanini GG, Serruys PW, Silber S, Khattab AA, van Geuns RJ, Richardt G et al (2011) The impact of patient and lesion complexity on clinical and angiographic outcomes after revascularization with zotarolimus- and everolimus-eluting stents: a substudy of the RESOLUTE All Comers Trial (a randomized comparison of a zotarolimus-eluting stent with an everolimuseluting stent for percutaneous coronary intervention). J Am Coll Cardiol 57(22):2221-2232

21. Morice MC, Serruys PW, Sousa JE, Fajadet J, Ban HE, Perin M et al (2002) A randomized comparison of a sirolimus-eluting stent with a standard stent for coronary revascularization. N Engl J Med 346(23):1773-1780

22. Schampaert E, Cohen EA, Schluter M, Reeves F, Traboulsi M, Title LM et al (2004) The Canadian study of the sirolimus-eluting stent in the treatment of patients with long de novo lesions in small native coronary arteries (C-SIRIUS). J Am Coll Cardiol 43(6):1110-1115

23. Fajadet J, Wijns W, Laarman GJ, Kuck KH, Ormiston J, Munzel T et al (2006) Randomized, double-blind, multicenter study of the endeavor zotarolimus-eluting phosphorylcholine-encapsulated stent for treatment of native coronary artery lesions: clinical and angiographic results of the ENDEAVOR II trial. Circulation 114(8):798-806 
24. Colombo A, Drzewiecki J, Banning A, Grube E, Hauptmann K, Silber S et al (2003) Randomized study to assess the effectiveness of slow- and moderate-release polymer-based paclitaxel-eluting stents for coronary artery lesions. Circulation 108(7):788-794

25. Kastrati A, Elezi S, Dirschinger J, Hadamitzky M, Neumann FJ, Schomig A (1999) Influence of lesion length on restenosis after coronary stent placement. Am J Cardiol 83(12):1617-1622

26. Hikichi Y, Inoue T, Node K (2009) Benefits and limitations of cypher stent-based bifurcation approaches: in vitro evaluation using micro-focus CT scan. J Interv Cardiol 22(2):128-134

27. Murasato Y, Hikichi Y, Horiuchi M (2009) Examination of stent deformation and gap formation after complex stenting of left main coronary artery bifurcations using microfocus computed tomography. J Interv Cardiol 22(2):135-144

28. Shiratori Y, Brugaletta S, Alvarez-Contreras L, Azpeitia Y, Ospino N, Gaido S, et al. (2013) One-year head to head comparison of the neointimal response between sirolimus eluting stent with reservoir technology and everolimus eluting stent: an optical coherence tomography study. Catheter Cardiovasc Interv 82(4):E428-E436

29. Gomez-Lara J, Brugaletta S, Farooq V, Onuma Y, Diletti R, Windecker $S$ et al (2011) Head-to-head comparison of the neointimal response between metallic and bioresorbable everolimuseluting scaffolds using optical coherence tomography. JACC Cardiovasc Interv 4(12):1271-1280

30. Kim SJ, Lee H, Cho JM, Park CB, Kim W, Kato K et al (2013) Comparison of zotarolimus-eluting stent and everolimus-eluting stent for vascular healing response: serial 3-month and 12-month optical coherence tomography study. Coron Artery Dis 24(5): 431-439

31. Gutierrez-Chico JL, van Geuns RJ, Regar E, van der Giessen WJ, Kelbaek H, Saunamaki K et al (2011) Tissue coverage of a hydrophilic polymer-coated zotarolimus-eluting stent vs. a fluoropolymer-coated everolimus-eluting stent at 13-month follow-up: an optical coherence tomography substudy from the RESOLUTE All Comers trial. Eur Heart J 32(19):2454-2463

32. Goto I, Itoh T, Kimura T, Fusazaki T, Matsui H, Sugawara S et al (2011) Morphological and quantitative analysis of vascular wall and neointimal hyperplasia after coronary stenting: comparison of bare-metal and sirolimus-eluting stents using optical coherence tomography. Circ J 75(7):1633-1640

33. Guagliumi G, Costa MA, Sirbu V, Musumeci G, Bezerra HG, Suzuki N et al (2011) Strut coverage and late malapposition with paclitaxel-eluting stents compared with bare metal stents in acute myocardial infarction: optical coherence tomography substudy of the Harmonizing Outcomes with Revascularization and Stents in Acute Myocardial Infarction (HORIZONS-AMI) Trial. Circulation 123(3):274-281

34. Foin N, Torii R, Alegria E, Sen S, Petraco R, Nijjer S et al (2013) Location of side branch access critically affects results in bifurcation stenting: insights from bench modeling and computational flow simulation. Int J Cardiol 168(4):3623-3628

35. Alegria-Barrero E, Foin N, Chan PH, Syrseloudis D, Lindsay AC, Dimopolous K et al (2012) Optical coherence tomography for guidance of distal cell recrossing in bifurcation stenting: choosing the right cell matters. EuroIntervention 8(2):205-213

36. Okamura T, Yamada J, Nao T, Suetomi T, Maeda T, Shiraishi K et al (2011) Three-dimensional optical coherence tomography assessment of coronary wire re-crossing position during bifurcation stenting. EuroIntervention 7(7):886-887

37. Gutierrez-Chico JL, Regar E, Nuesch E, Okamura T, Wykrzykowska J, Di MC et al (2011) Delayed coverage in malapposed and side-branch struts with respect to well-apposed struts in drug-eluting stents: in vivo assessment with optical coherence tomography. Circulation 124(5):612-623 